\title{
Partitioning behavior, source identification, and risk assessment of perfluorinated compounds in an industry-influenced river
}

\author{
Jiapei Lv', Changsheng Guo', Shuxuan Liang ${ }^{2}$, Yuan Zhang ${ }^{1}$ and Jian X u $^{{ }^{*}}$ (I)
}

\begin{abstract}
Background: The widespread application of perfluorinated compounds (PFCs) makes them ubiquitously distributed in the environment. Investigation of contamination profiles, distribution, possible sources and risks of PFCs in Liaohe River, an industry-influenced river in northeast China was conducted in the present study.

Results: The total PFCs concentrations ( $\sum$ PFCs) were in the range $0.38-127.88 \mathrm{ng} / \mathrm{L}$ (average value of $36.41 \mathrm{ng} / \mathrm{L}$ ) in water and $1.72-10.44 \mathrm{ng} / \mathrm{g}$ dry weight (average of $4.99 \mathrm{ng} / \mathrm{g}$ ) in sediment. Perfluorooctanoic acid (PFOA) was the dominant individual in water and sediment phases, in the range $0.38-73.94 \mathrm{ng} / \mathrm{L}$ in water and below detection limit $(\mathrm{BDL})$ to $7.88 \mathrm{ng} / \mathrm{g} \mathrm{dw}$ in the sediments. The organic carbon normalized partition coefficients $\left(K_{\text {oc }}\right)$ ranged from $2.46 \mathrm{~L} /$ $\mathrm{kg}$ (PFHXA) to $4.29 \mathrm{~L} / \mathrm{kg}$ (PFUnDA). The average $K_{\text {oc }}$ values for perfluorocarboxylic acids (PFCAs) increased by 0.13-0.62 log unit with each increasing $\mathrm{CF}_{2}$ moiety, and the $K_{\mathrm{oc}}$ values were lower than perfluoroalkyl sulfonates (PFASs). The sources of PFCs identified from diagnostic ratios suggested that the contaminants were mainly from the emission of manufacturing processes and precursors degradation. Result from risk assessment indicated that the immediate health impact through intaking water was negligible, but the levels of PFOA in surface water might cause effects on aquatic ecosystem. The mass inventories of $\sum$ PFCs and PFOA were estimated to be $328.74 t$ and $103.43 \mathrm{t}$ in the study area, respectively, suggesting that the sediment in Liaohe River may act as a potential PFCs source to the surrounding areas.
\end{abstract}

Conclusion: This study demonstrated that PFCs were widely presented in the water and sediments of Liaohe River. In general, PFCAs had higher concentrations and detection frequency than PFSAs, and PFCAs with short carbon chains had much higher detection frequencies than long-carbon chain compounds in water. Among all PFC homologues, the average $K_{\text {oc }}$ increased with the increased carbon chain. $K_{\text {oc }}$ values were higher for PFSAs than PFCAs. The risk assessment suggested that PFOA in water may exert adverse effect on the aquatic ecosystem. Liaohe River was likely acting as a PFCs source to the surrounding area.

Keywords: Perfluorinated compounds, Distribution, Source, Risks, Inventory, Liaohe River

\section{Background}

Perfluorinated compounds (PFCs) have been widely used as surfactants, surface protectors and performance chemicals in fire-fighting foams, leather, food packing and shampoos [1], due to their high surface activities, thermal

\footnotetext{
*Correspondence: xujian@craes.org.cn

'State Key Laboratory of Environmental Criteria and Risk Assessment, Chinese Research Academy of Environmental Sciences, Beijing 100012, China

Full list of author information is available at the end of the article
}

and chemical resistances, and hydrophilic and hydrophobic properties [2]. The widespread application of PFCs makes them ubiquitously distributed in the environment [3], such as in the air [4], rivers [5], lakes [6], and even in remote areas (Antarctic and Arctic areas) [7]. Previous studies revealed PFCs could accumulate in biota, including fish [8], invertebrates [9], birds [10], marine mammal [11], and human bodies [12], and magnify along the food webs [13]. They could exert adverse effect to aquatic and terrestrial organisms. Toxicological studies indicated 
that adverse effect in organisms and their offspring was observed when exposed to PFCs during embryonic stage. Perfluorooctane sulfonate (PFOS) together with its salts and perfluorooctane sulfonyl fluoride (POSF) has been listed under the Stockholm Convention as the persistent organic pollutants in 2009.

In 2003, POSF began to be produced in China and used in commercial products, with the annual production less than 100 tons. From 2004 to 2009, the annual production grew steadily, with the annual production of 91, 165, $247,195,250,300$ tons, respectively, among which half was consumed domestically $[14,15]$. The cumulative historical production volume of PFOS and related chemicals was estimated to be 1800 tons by 2011 .

Due to the intrinsic properties of most PFCs such as moderate water solubility and low vapor pressure, PFCs tend to finally accumulate to water and sediments in the aquatic ecosystem $[10,16]$. Ubiquitous detection of PFCs has been reported in different regions of Chinese water environments. Primary sources of PFCs in the aquatic environment included atmospheric precipitation, emission from manufacturing process [17], the precursor transformation, effluents of treated wastewater [18] and street runoff [19].

Liaohe River is located in northeast China, and it runs across areas with petrochemical industry, steel factories, pharmacy, machinery, and building materials [20], and finally enters Bohai Sea [21]. In recent years, the water quality of Liaohe River continuously decreased because of the rapid population growth and the increasing industrial and agricultural activities [22]. Many contaminants have been detected in Liaohe River including polycyclic aromatic hydrocarbons [23], organochlorine pesticides [24], polychlorinated dibenzo- $p$-dioxins and dibenzofurans, polychlorinated biphenyls [25], and short chain chlorinated paraffins [26]. Previous study also showed that PFCs were detected in Liaohe River, with concentrations in the range of $<\mathrm{LOQ}$ to $31 \mathrm{ng} / \mathrm{L}$. PFOA and PFOS were the dominant PFCs, with mean concentrations of 27 and $4.7 \mathrm{ng} / \mathrm{L}$, respectively [15]. The occurrence of PFCs in sediments of Daliao River system was also reported in the range of 0.29 to $1.03 \mathrm{ng} / \mathrm{g}$, dominated by PFOA and PFOS [3]. To the best of our knowledge, information is scarce on the PFCs source characterization, health risks, and their inventory in this area.

This paper was focused on the occurrence of PFCs in surface water and sediments in Liaohe River. The measured data allowed us to evaluate the partitioning behavior, the preliminary estimation of PFCs inventories and the screening-level ecological risk assessment for PFCs in the aquatic environment. Possible pollution sources of PFCs were also discussed.

\section{Materials and methods}

\section{Sampling area and sample collection}

Liaohe River includes Liao River, Daliao River, Hun River and Taizi River (Fig. 1). Fifty sites were selected from ten rivers (Liao River, Hun River, Taizi River, Daliao River and their tributaries including Qing River, Chai River, Xiushui River, Yangximu River, Liu River, and Shahe River) for collecting surface water and sediments in October 2012.

A clean stainless steel bucket was used to collect surface water, which was stored in polypropylene (PP) bottles. The stainless steel grab sampler was used to collect surface sediments which were placed in PP bags. All equipments were rinsed by methanol and Milli-Q water before use.

\section{Chemicals and reagents}

Perfluorobutanoate (PFBA), perfluoropentanoate (PFPA), perfluorododecanoate (PFDoDA), perfluorotridecanoate (PFTrDA), perfluorooctane sulfonamide (PFOSA), perfluorodecane sulfonate (PFDS), perfluorooctane sulfonate (PFOS) and perfluorobutane sulfonate (PFBS) were obtained from Sigma-Aldrich (St. Louis, MO, USA). Perfluoroundecanoate (PFUnDA), perfluoroheptanoate (PFHpA) and perfluorohexanoate (PFHxA) were obtained from Matrix Scientific (Columbia, SC, USA). Perfluorononanoate (PFNA), perfluorooctanoate (PFOA), perfluorodecanoate (PFDA), potassium salts of perfluorohexane sulfonate (PFHxS), perfluoro- $n-\left[{ }^{13} \mathrm{C}_{8}\right]$ octanoic acid $\left({ }^{13} \mathrm{C}_{8}\right.$ PFOA) and sodium perfluoro-1-[1,2,3,4- $\left.{ }^{13} \mathrm{C}_{4}\right]$ octanesulfonate $\left({ }^{13} \mathrm{C}_{4}\right.$-PFOS) were obtained from Wellington Laboratories (Guelph, Ontario, Canada). Solvents including methanol and $\mathrm{NH}_{4} \mathrm{OH}$ were HPLC grade from Beijing Chemical Factory (Beijing, China). All stock solutions were prepared in methanol and stored in polypropylene (PP) tubes or vials at $4{ }^{\circ} \mathrm{C}$.

\section{Extraction, cleanup and analysis}

The pretreatment of water and sediment samples were described elsewhere [27]. In brief, 1 L water sample spiked with 5 ng surrogate standard was extracted with Oasis WAX-SPE cartridge (6 cc, $150 \mathrm{mg}$; Waters Corp. Milford, USA). Prior to water loading, the cartridges were pre-conditioned with $4 \mathrm{~mL} 0.5 \% \mathrm{NH}_{4} \mathrm{OH} / \mathrm{CH}_{3} \mathrm{OH}$ (v/v), $4 \mathrm{~mL}$ methanol and $4 \mathrm{~mL}$ Milli-Q water. The cartridge was then vacuum-dried for $2 \mathrm{~h}$. Elution was carried out with $4 \mathrm{~mL}$ methanol and $4 \mathrm{~mL} 0.5 \% \mathrm{NH}_{4} \mathrm{OH} /$ $\mathrm{CH}_{3} \mathrm{OH}(\mathrm{v} / \mathrm{v})$ with gravity. The eluate was then concentrated to $1 \mathrm{~mL}$ for instrumental analysis.

Sediments were sonic extracted by methanol. Generally, two grams of homogenized freeze-dried samples were weighted and transferred into $50 \mathrm{~mL}$ PP centrifuge tubes and spiked with $5 \mathrm{ng}$ surrogate standard. The tubes 


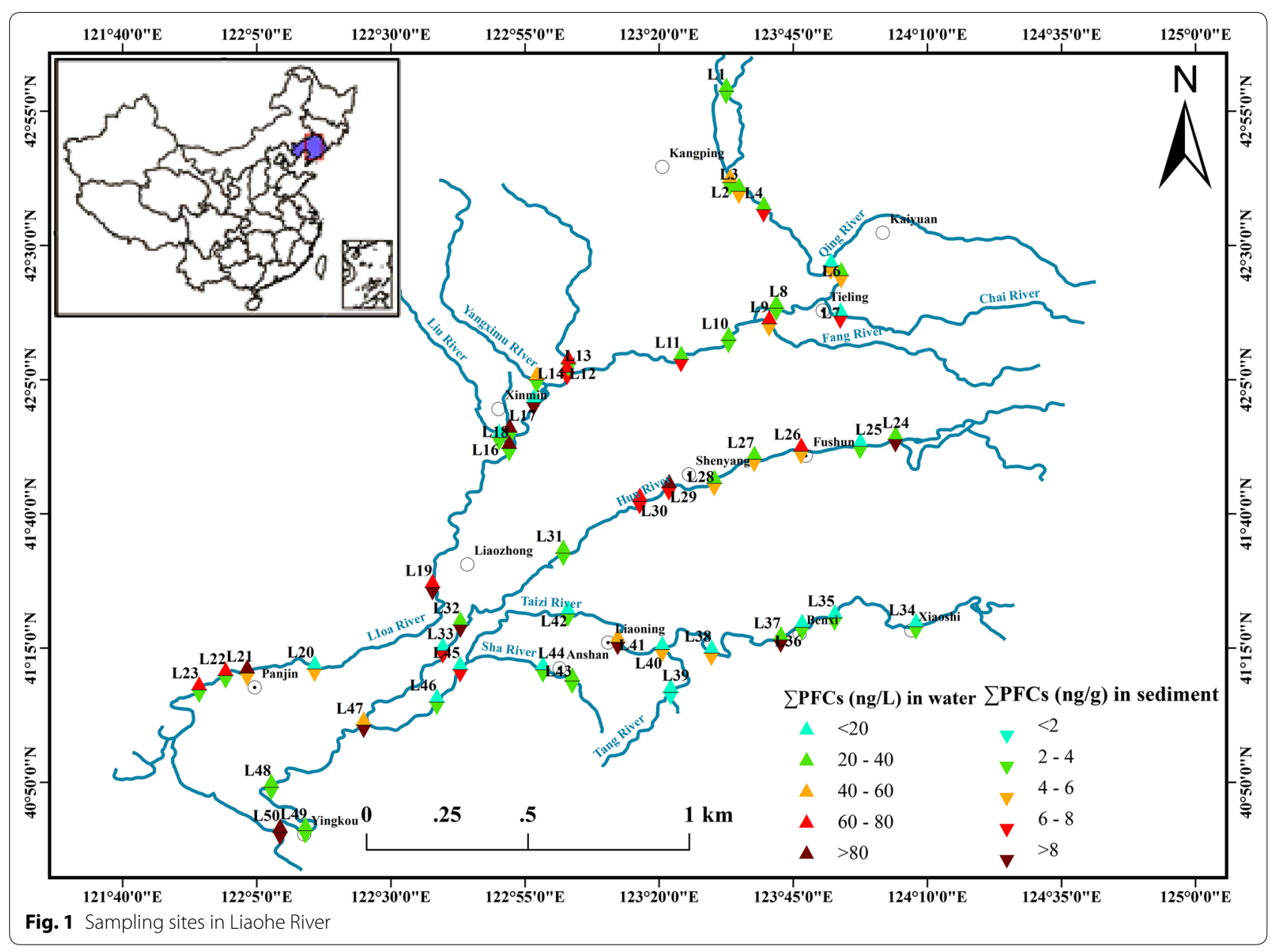

were added with $8 \mathrm{~mL}$ of methanol and shaken for $20 \mathrm{~min}$ at the speed of $250 \mathrm{rpm}$ at $25{ }^{\circ} \mathrm{C}$, sonicated for $10 \mathrm{~min}$, and then centrifuged at $8000 \mathrm{rpm}$ for $10 \mathrm{~min}$. After centrifugation, the supernatant was collected and decanted to a clean beaker. The extraction was repeated 2 more times, and the extracts were combined. The extracts were diluted to $200 \mathrm{~mL}$ with ultrapure water, and passed through Oasis WAX-SPE cartridges at a rate of $3 \mathrm{~mL} /$ min. The analytes were then eluted with $4 \mathrm{~mL}$ methanol and $4 \mathrm{~mL} 0.5 \% \mathrm{NH}_{4} \mathrm{OH} / \mathrm{CH}_{3} \mathrm{OH}(\mathrm{v} / \mathrm{v})$. The extract was blown down to $1 \mathrm{~mL}$ under a nitrogen stream and transferred into GC vials for analysis.

\section{Instrumental analysis}

The target compounds were quantified with an Agilent 1200 LC equipped with a ZORBAX Eclipse XDBC18 column $(2.1 \times 100 \mathrm{~mm}, 5 \mu \mathrm{m})$ and a $6410 \mathrm{~A}$ mass spectrometer (Agilent, Palo Alto, CA). The column was maintained at $40{ }^{\circ} \mathrm{C}$ during the sample analysis. The mobile phase consisted of eluent A (methanol) and eluent $\mathrm{B}$ (2.5 $\mathrm{mM}$ ammonium acetate solution). Flow rate was kept at $0.25 \mathrm{~mL} / \mathrm{min}$, and the injection volume was $20 \mu \mathrm{L}$. The separation of PFCs was achieved with a gradient program, with an initial gradient of $30 \% \mathrm{~A}$, increased to $40 \% \mathrm{~A}$ at $4 \mathrm{~min}$, and continuously increased to $90 \% \mathrm{~A}$ at $9 \mathrm{~min}$. The gradient was reverted to $30 \% \mathrm{~A}$ at $13 \mathrm{~min}$ and maintained for $2 \mathrm{~min}$. The operating parameters for LC instrumental analysis could refer to the previous publication, and the MS/MS parameters for optimizing the instrument were the same as our previous study [27].

Analytes were identified with multiple responses monitoring analysis. Internal calibration was used to quantify analytes. ${ }^{13} \mathrm{C}_{8}$-PFOA was the internal standard for PFCA and ${ }^{13} \mathrm{C}_{4}$-PFOS for PFOS, respectively. The analytical process was conducted in triplicate to evaluate the precision.

\section{Quality assurance and quality control (QA/QC)}

During the experimental processes, the polytetrafluoroethene (PTFE) and fluoropolymer materials were avoided to reduce the background contamination. The recoveries of the target compounds were determined by subtraction 
of the background concentrations from the detected spiking concentration of the compounds in the test samples. Quantification of each PFCs compound was obtained using the internal standard method. Calibration curves were constructed in the range of $0.2 \mu \mathrm{g} / \mathrm{L}$ to $50 \mu \mathrm{g} / \mathrm{L}$ for the PFCs, with linearity $r^{2}>0.995$. The mean recoveries of PFCs spiked into water and sediment samples were from 61 to $118 \%$ for water, and from 69 to $128 \%$ for sediment, respectively. Matrix recovery test was carried out in triplicate, and the relative standard deviations were below $18 \%$. The LOQs were determined by the injection of spiked water extracts $(n=3)$ and estimated as the minimum detectable amount of analyte with a signal-to-noise ratio of $10: 1$ and the deviations were within $\pm 20 \%$ from theoretical values of the triplicate injection. The LOQs for each compound ranged from 0.10 to $0.60 \mathrm{ng} / \mathrm{L}$ in water, and 0.02 to $0.10 \mathrm{ng} / \mathrm{g}$ in sediment.

\section{Results and discussion}

Distribution of PFCs in surface water and sediments

The aqueous concentrations of PFCs in Liaohe River were shown in Fig. 2. A total of 15 PFCs including C4C13 perfluorocarboxylic acids (PFCA) (e.g. PFBA,
PFPA, PFHxA, PFOA, PFHpA, PFNA, PFUnDA, PFDA, PFDoDA and PFTrDA), C4, C6, C8 and C10 perfluoroalkyl sulfonates (PFASs) (e.g. PFHxS, PFBS, PFOS and PFDS) and one non-ionic PFC (PFOSA) were quantified. The $\sum$ PFCs concentration in the water phase ranged between 0.38 and $127.88 \mathrm{ng} / \mathrm{L}$, with the mean concentration value of $36.41 \mathrm{ng} / \mathrm{L}$. The concentrations of PFCAs and PFASs were in the range of 0.38 to $127.88 \mathrm{ng} / \mathrm{L}$ and below detection limit (BDL) to $55.96 \mathrm{ng} / \mathrm{L}$, respectively.

Geographically, the spatial distribution of PFCs was associated with urbanization and industrialization. The average value of $\sum$ PFCs (the mean concentrations of 15 PFCs in this study) of Daliao River $(51.01 \mathrm{ng} / \mathrm{L})$ was higher than the other three rivers $(44.79 \mathrm{ng} / \mathrm{L}$ for Liao River, $41.05 \mathrm{ng} / \mathrm{L}$ for Hun River and $13.52 \mathrm{ng} / \mathrm{L}$ for Taizi River). Of all the sampling sites, site H29 in Hun River presented the highest $\sum$ PFCs up to $127.88 \mathrm{ng} / \mathrm{L}$, followed by D50 in Daliao River (97.75 $\mathrm{ng} / \mathrm{L})$ and L17 in Liao River $(89.70 \mathrm{ng} / \mathrm{L})$. It is obvious that human activities affected the PFCs distribution in the water samples. For instance, $\mathrm{PPFC}$ in sites L1, L3-L7 in the upstream of Liao River were lower than in the midstream and downstream of the river, where wastewater from the

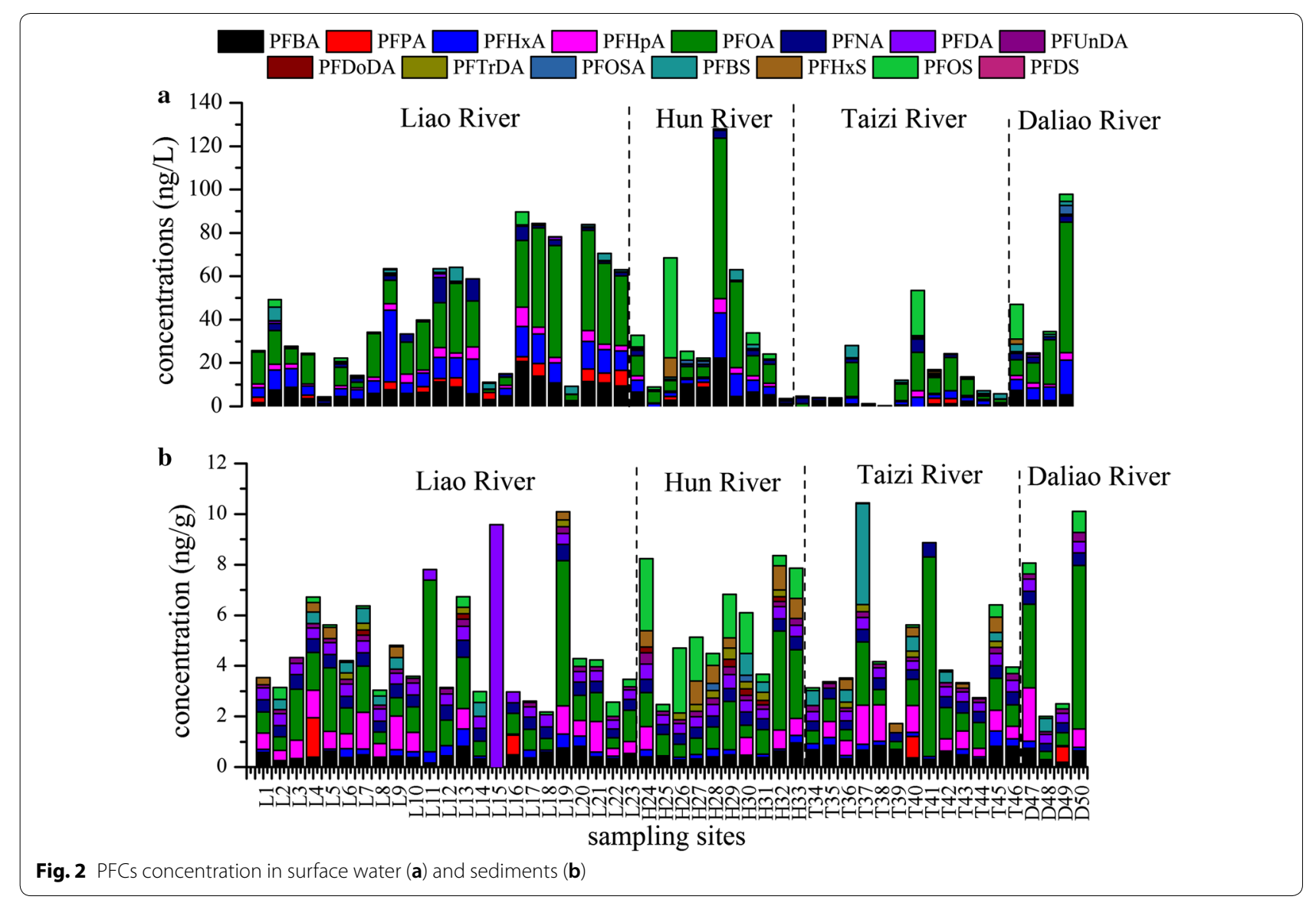


surrounding cities, such as Xinmin City (L12-L14, L17L18), Liaozhong City (L19), and Panjin City (L21-L23) was discharged into the river water. Similarly, in Hun River, the low $\sum$ PFCs at $\mathrm{H} 24$ and $\mathrm{H} 25$ were resulted from their locations close to Dahuofang Reservoir which was the drinking water source for Fushun City and surrounding districts, whereas high PFCs concentrations were detected at sites adjacent to Shenyang City (H29 and H30) and Fushun City (H26). This was similar to the results reported by Sun et al. [28], who found that high PFCs concentrations were detected in highly industrialized districts in Hun River. For instance, Shenyang City and Fushun City contributed significant loads of PFCs to the Hun River. The PFCs contribution from Shenyang City was obviously greater than those from Fushun City, which could be explained by the population and economic level. The PFCs concentration in the upstream of the Hun River was quite low. Generally, the PFCs concentrations showed geographical variations. Industrial and municipal cities were characterized by higher contamination concentrations. Similarly, a research estimating the emission of PFOS in China found that most intensively industrialized regions on eastern coastal areas showed higher emission rates and density of PFOS than those in western and northern China [15].

Relatively low PFCs concentrations were determined in Taizi River, except for site T41 which was close to Liaoyang City. Domestic sewage and industrial wastewater from these cities influenced the $\sum$ PFCs level in the river water. Low PFC levels in Taizi River were mainly due to the low anthropogenic activities in the river basin.

In general, the PFCAs had higher concentrations and detection frequency than PFASs. The detection frequencies of short-carbon chain PFCAs (PFBA, PFHxA, PFHpA, PFOA and PFNA) were much higher than longcarbon chain compounds (PFDA, PFUnDA, PFDoDA and PFTrDA). Figure 3 showed that PFOA was the predominant pollutant in the river water. PFOA was reported to be the intermediate compound during the other PFCs synthesis and the final metabolite or degradation product of some related compounds [29]. This result was consistent with previous studies such as in Pearl River Delta region [30], Liaodong Bay [31], Haihe River Basin [32], and Yangtze River Delta [33].

The $\sum$ PFCs in sediments in Liaohe River ranged from 1.72 to $10.44 \mathrm{ng} / \mathrm{g}$, with an average concentration of $4.99 \mathrm{ng} / \mathrm{g}$. The highest $\sum \mathrm{PFCs}$ was found in T37 (10.44 ng/g), followed by D50 (10.11 ng/g) and L19 (10.09 ng/g). Generally, Hun River (mean value of $5.78 \mathrm{ng} / \mathrm{g}$ ) and Daliao River (mean $5.67 \mathrm{ng} / \mathrm{g}$ ) were more polluted than Liao River (mean $4.70 \mathrm{ng} / \mathrm{g}$ ) and Taizi River (mean $4.70 \mathrm{ng} / \mathrm{g}$ ). The predominant compound PFOA ranged from $\mathrm{BDL}$ to $7.88 \mathrm{ng} / \mathrm{g}$, with the average contribution of $28.03 \%$ to $\sum$ PFCs. In contrast to the water samples, the PFCs showed a different compositional profile. Long carbon chain PFCs such as PFDA, PFUnDA, PFDoDA and PFTrDA were observed in sediment samples with high concentration and detection frequencies, indicating that long chain PFCs were tending to partition into sediment.

\section{Partitioning of PFCs between water and sediments}

Partitioning is an important process in the fate and transport of pollutants in the aquatic environment. The sediment-water partitioning coefficients $\left(K_{\mathrm{d}}\right)$ were determined using $K_{\mathrm{d}}=C_{\mathrm{s}} / C_{\mathrm{w}}$, where $C_{\mathrm{s}}$ and $C_{\mathrm{w}}$ are the PFCs concentrations in sediments $(\mathrm{ng} / \mathrm{kg})$ and water $(\mathrm{ng} / \mathrm{L})$, respectively. The organic carbon normalized adsorption coefficient $\left(K_{\mathrm{oc}}\right)$ was calculated by $K_{\mathrm{oc}}=K_{\mathrm{d}} / f_{\text {oc }}$, where $f_{\text {oc }}$ is the sediment organic carbon fraction.

Different $K_{\mathrm{oc}}$ and $K_{\mathrm{d}}$ values reflected different sorption potentials and partition of PFCs in riverine environment. The average $\log K_{\mathrm{d}}$ of PFCs in Liaohe River were in the range of 1.58 (PFHxA) to 3.14 (PFUnDA), and average $\log K_{\mathrm{oc}}$ ranging from 2.46 (PFHxA) to 4.29 (PFUnDA) (Table 1). The $\log K_{\mathrm{oc}}$ value of PFOS $(3.33 \pm 0.74)$ in this study was consistent with the laboratory experiment result (3.7 \pm 0.56$)$, but $\log K_{\mathrm{oc}}$ of PFOA showed a discrepancy $(3.07 \pm 0.63$ vs. $2.4 \pm 0.12)$. The $\log K_{\mathrm{d}}$ and $\log K_{\text {oc }}$ values obtained in the present study were similar to those in Haihe River [32], but significantly different from those in aquatic systems in the Netherlands [34] and Tangxun Lake [35]. The field-based partitioning values may be influenced by many factors, such as the composition and property of sediment and water. Earlier researches proposed that sediment and water conditions (such as $\mathrm{pH}$, aqueous $\mathrm{Ca}^{2+}$ and salinity) were significantly correlated with their sorption [36]. Similar correlations were found in this study as shown in Table 2.

Among the PFCs homologues, the average $K_{\text {oc }}$ generally increased with the increasing carbon chain. As shown in Table 1, the values followed the order of PFHxA $<$ PFHpA $<$ PFOA $<$ PFNA $<$ PFDA $<$ PFUnDA, increasing by $0.13-$ $0.62 \log$ units with each $\mathrm{CF}_{2}$ moiety. Similarly, in Haihe River, the average $\log K_{\mathrm{oc}}$ ranged from 3.3 for PFHpA to 4.4 for PFUnDA, increasing by $0.1-0.6 \log$ units with each $\mathrm{CF}_{2}$ moiety [32]. Previous research showed that the hydrophobic interaction was the main factor affecting the sediment-water partition of C8-C12 PFCAs and C6, C8 PFASs, and with the increasing perfluoroalkyl chain length the sediment sorption potential increased [35]. It confirmed that the perfluorocarbon chain length was the dominant structure feature affecting the PFCs sorption. Interestingly, the slope of the regression of PFCAs $\log K_{\text {oc }}$ versus perfluoroalkyl chain length (Fig. 4) was $0.39\left(R^{2}=0.97\right)$, which was slightly lower than that from 


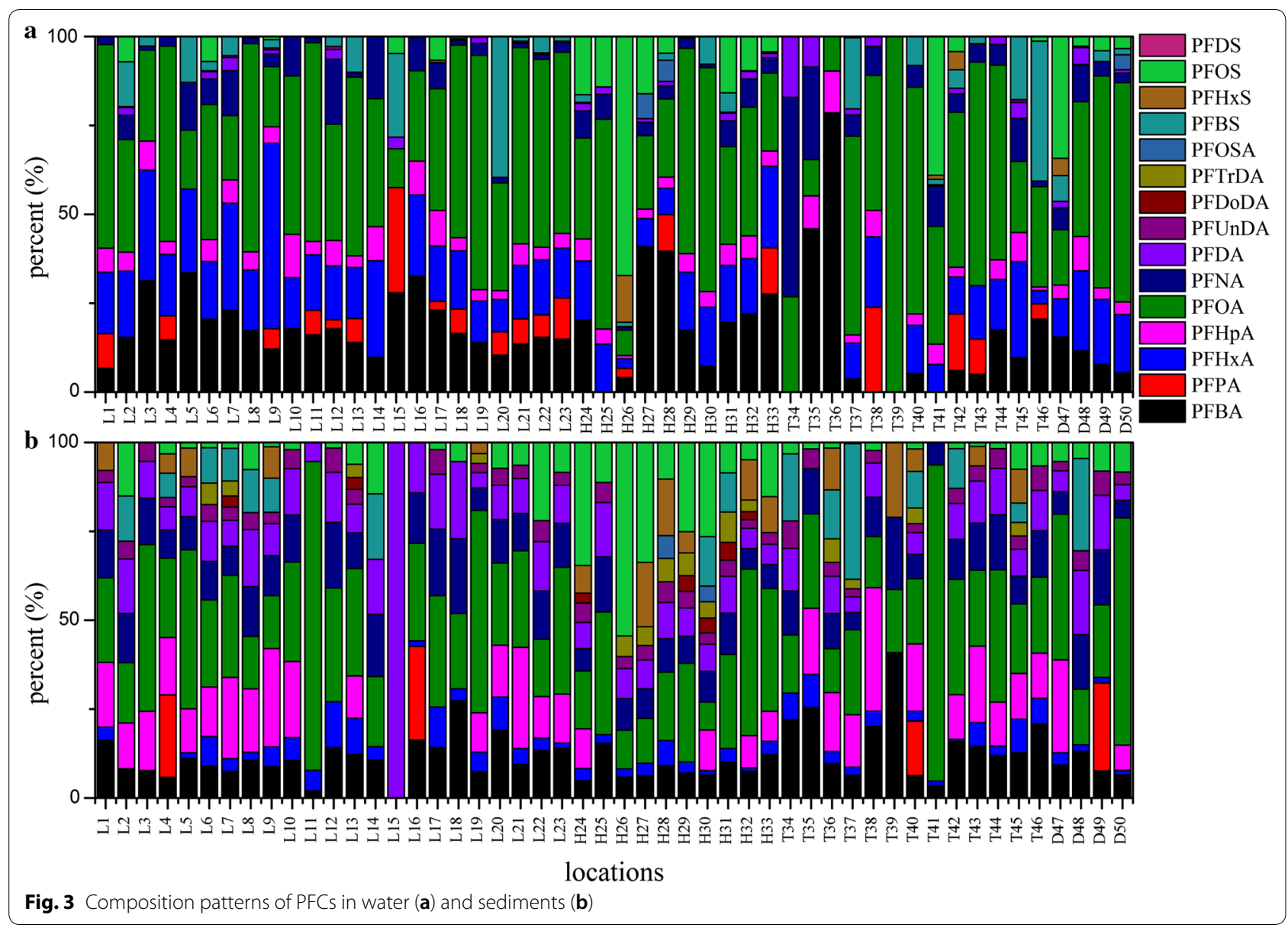

Table $1 \log K_{d}$ and $\log K_{o c}$ values of PFCs from Liaohe River

\begin{tabular}{|c|c|c|c|c|c|c|}
\hline \multirow[t]{2}{*}{ Compound } & \multicolumn{2}{|l|}{ This study } & \multicolumn{2}{|c|}{ Haihe River, China [32] } & \multirow{2}{*}{$\begin{array}{l}\text { Aquatic system, } \\
\text { Netherlands [34] } \\
\log K_{\mathrm{d}}\end{array}$} & \multirow{2}{*}{$\begin{array}{l}\text { Tangxun } \\
\text { Lake, China } \\
\text { [35] } \\
\log K_{\text {oc }}\end{array}$} \\
\hline & $\log K_{\mathrm{d}}$ & $\log K_{\mathrm{oc}}$ & $\log K_{\mathrm{d}}$ & $\log K_{\mathrm{oc}}$ & & \\
\hline PFBA & $2.03 \pm 0.49$ & $3.01 \pm 0.51$ & $-^{\mathrm{a}}$ & - & - & $2.5^{b}$ \\
\hline PFHxA & $1.58 \pm 0.60$ & $2.46 \pm 0.63$ & $2.2 \pm 0.4$ & $3.4 \pm 0.4$ & - & - \\
\hline PFHpA & $2.06 \pm 0.53$ & $2.75 \pm 0.53$ & $2.0 \pm 0.5$ & $3.3 \pm 0.5$ & - & 1.9 \\
\hline PFOA & $2.14 \pm 0.64$ & $3.07 \pm 0.63$ & $2.1 \pm 0.4$ & $3.4 \pm 0.4$ & $1.83 \pm 0.40$ & $2.3 \pm 0.23$ \\
\hline PFNA & $2.57 \pm 0.47$ & $3.54 \pm 0.51$ & $2.3 \pm 0.4$ & $3.6 \pm 0.4$ & $2.89 \pm 0.53$ & - \\
\hline PFDA & $3.12 \pm 0.46$ & $4.16 \pm 0.56$ & $2.9 \pm 0.4$ & $4.2 \pm 0.3$ & $2.87 \pm 0.23$ & - \\
\hline PFUnDA & $3.14 \pm 0.45$ & $4.29 \pm 0.55$ & 3.1 & $4.4 \pm 0.3$ & - & - \\
\hline PFBS & $2.54 \pm 0.35$ & $3.54 \pm 0.19$ & $3.2 \pm 0.2$ & $4.4 \pm 0.3$ & $1.42 \pm 0.50$ & - \\
\hline PFOS & $2.31 \pm 0.71$ & $3.33 \pm 0.74$ & - & - & $2.35 \pm 0.35$ & $3.7 \pm 0.38$ \\
\hline
\end{tabular}

\footnotetext{
${ }^{a}$ Not obtained
}

b Average value

Kwadijk et al's [34] and Higgins and Luthy's reports [37]. The difference was mainly affected by the different PFC homologues in different studies.

\section{Potential source identification}

The diagnostic ratios, such as PFOS/PFOA, PFOA/PFNA and PFHpA/PFOA, have been applied to identify the 
Table 2 Pearson correlation analysis for the water quality parameters and $K_{\mathrm{oc}}$ values in the water samples collected from the Liaohe River

\begin{tabular}{llllcrrr}
\hline $\log \boldsymbol{K}_{\text {oc }}$ & T & DO & $\begin{array}{l}\text { Electrical } \\
\text { conductivity }\end{array}$ & TDS & Salinity & ORP & pH \\
\hline PFBA & -0.089 & -0.159 & 0.028 & 0.107 & 0.168 & -0.168 & -0.138 \\
PFHxA & -0.195 & 0.002 & $-0.317^{*}$ & -0.22 & -0.146 & 0.067 & 0.031 \\
PFHpA & -0.124 & -0.154 & -0.345 & -0.223 & -0.323 & 0.1 & -0.123 \\
PFOA & -0.19 & -0.033 & $-0.454^{* *}$ & $-0.387^{* *}$ & $-0.337^{*}$ & 0.234 & -0.092 \\
PFNA & 0.028 & -0.175 & -0.01 & 0 & 0.045 & -0.03 & 0.041 \\
PFDA & 0.146 & -0.18 & 0.015 & -0.088 & -0.039 & -0.078 & -0.06 \\
PFUnDA & -0.317 & -0.242 & 0.047 & 0.255 & 0.274 & 0.449 & $-0.613^{* *}$ \\
PFBS & -0.442 & 0.152 & -0.576 & -0.571 & -0.355 & 0.463 & 0.127 \\
PFOS & -0.214 & $-0.456^{*}$ & 0.069 & 0.246 & 0.136 & 0.075 & -0.153 \\
\hline
\end{tabular}

${ }^{*} p<0.05,{ }^{* *} p<0.01$

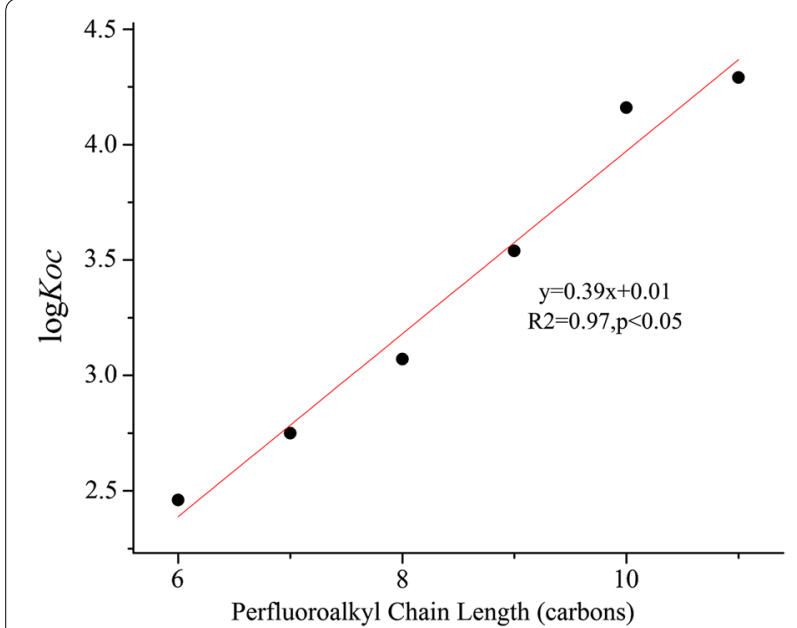

Fig. 4 Correlation between the mean $\log K_{o c}$ and the perfluoroalkyl chain length

potential sources of PFCs in the environment. In most of the water samples in this study, PFOA showed higher concentration than PFOS, and the ratios of PFOS/PFOA were generally less than 1.0. In several sampling sites, such as H26 (9.57) from Fushun, T41 (1.18) from Liaoyang and D47 (2.20) which was the confluence of Taizi River and Hun River, higher PFOS concentrations were observed than PFOA, indicating of potential PFOS point sources. It should be stated that above mentioned sites were close to petrochemical industrial areas. Ratios of PFOS/PFOA calculated in this study were consistent with findings in surface waters from northern China [38], the Tennessee River and Lake Michigan in USA [16] and 20 wastewater treatment plants effluents in 11 cities in economically developed areas of China [39].
PFHpA was found in most water samples with a detection frequency of $90 \%$, and it was also the dominant individual in some sampling sites. Several researches have reported the dominance of this compound in surface water. Simcik and Dorweiler [40] proposed that relatively high concentrations of $\mathrm{PFHpA}$ were due to the atmospheric deposition. The ratio of PFHpA/PFOA was, therefore, used as a tracer of atmospheric deposition. The ratios of PFHpA/PFOA in this study was less than 1, but at T36 from Benxi City the ratio was 1.22, suggesting that atmosphere deposition may not be the major source of PFCs in the study area, and T36 might be affected by point-source pollution as a result of industrialization.

The ratio of PFOA/PFNA in the range of 7 to 15 indicated the direct emission of manufacturing process [41]. The ratios of PFOA/PFNA in this study were in the range of 0.39 to 67.43. The sites with ratios for PFOA/PFNA in the range of 7-15 were mostly adjacent to industrial cities, such as Fushun [e.g. H25 (8.44), H26 (8.62)], Benxi [e.g. T37 (9.34)], Liaoyang [e.g. T42 (8.45)], Anshan [e.g. T43 (12.53), T44 (9.43)], Yingkou [e.g. D49 (14.79)], verifying that manufacturing processes did influence the PFCs levels in the study area. High ratios of PFOA/PFNA also indicated the degradation of precursors. Thus, the PFCs in Liaohe River were from the emission of manufacturing processes and precursors degradation.

\section{Risk assessment}

In this study, the assessment of risks to aquatic biota exposed to PFCs was conducted based on the risk quotient (RQ) method, which was determined by comparing the detected concentrations of the target individuals with the advisory guideline values. The RQ values greater than 1 indicate that the water could pose a risk to aquatic ecosystem. In this study, the risk of three indicator 
individuals, PFBS, PFOS and PFOA was assessed, with their environmental quality standards (EQS) shown in Table 3. As there were different environmental quality standards in different areas, the strictest standards were used in this study. All RQ values of PFBA, PFPA, PFHXA, PFNA, PFDA, PFBS, and PFOS were below 1, indicating that no risk existed to the aquatic organisms in Liaohe River. However, the RQs of PFOA in several sites were higher than 1 , suggesting that PFOA in surface water may exert adverse effect to the aquatic ecosystem. Even so, the risk of PFCs contamination in surface water to ecosystem should get more attention because of their bioaccumulation.

Liaohe River is the drinking water source of the region's population. For instance, Dahuofang Reservoir located in the upstream of Hun River serves as the drinking water source for Shenyang City, Fushun City, and Anshan City. Guanyinge Reservior and Caowo Reservior in the upstream and midstream of Taizi River are the drinking water source of downstream region. So the hazard ratio (HR) was used to estimate the potential health risk of PFOA and PFOS through water intake for biota through reference dose (RD) [27]:

$$
\begin{aligned}
& \mathrm{HR}_{\text {water }}=\mathrm{ADC}_{\text {water }} / \mathrm{RD}_{\text {water }} \\
& \mathrm{ADC}_{\text {water }}=C_{\text {water }} \times V_{\text {water }} \times 10^{-3} / \mathrm{W},
\end{aligned}
$$

where $\mathrm{ADC}_{\text {water }}$ is the estimated average daily consumptions $(\mu \mathrm{g} /(\mathrm{kg} \mathrm{d})), C_{\text {water }}$ represents the PFOA or PFOS concentration in water $(\mathrm{ng} / \mathrm{L}), V_{\text {water }}$ is the volume of drinking water for an adult each day (L), $W$ is the average weight of an adult $(\mathrm{kg})$. $R D_{\text {water }}$ is $0.333 \mu \mathrm{g} /(\mathrm{kg} \mathrm{d})$ for PFOA and $0.025 \mu \mathrm{g} /(\mathrm{kg} \mathrm{d})$ for PFOS. With the $\mathrm{HR}_{\text {water }}$ value below 1, the PFCs could not exert emerging health effects to biota. In this study, the adult average weight was assumed as $60 \mathrm{~kg}$ and the volume of water intake was $2 \mathrm{~L}$ each day. According to the concentration of PFOA

Table 3 Estimated risk quotients of 6 PFCs in surface water in Liaohe River

\begin{tabular}{llrl}
\hline Compound & $\begin{array}{l}\text { Range of concentrations } \\
\text { in surface water }(\mathbf{n g} / \mathbf{L})\end{array}$ & $\mathrm{EQS}^{\mathbf{a}}$ (ng/L) & RQ \\
\hline PFBA & n.d-22.26 & 1400 & $0-0.0159$ \\
PFPA & n.d-7.26 & 600 & $0-0.0121$ \\
PFHXA & n.d-33.14 & 200 & $0-0.1657$ \\
PFOA & $0.37-73.94$ & 20 & $0.01889-3.3971$ \\
PFNA & n.d-11.56 & 100,000 & $0-0.0001$ \\
PFDA & n.d-1.76 & 11,000 & $0-0.0001$ \\
PFBS & n.d-6.44 & 600 & $0-0.0107$ \\
PFOS & n.d-46.12 & 50 & $0-0.9223$
\end{tabular}

a EQS of PFBA, PFPA, PFHXA, PFOA, PFBS were obtained from Valsecchi et al. [42]; EQS of PFOS was obtained from Brooke et al. [43]; EQS of PFNA and PFDA were obtained from Hoke et al. [44] and PFOS in Liaohe River, the highest $\mathrm{HR}_{\text {water }}$ value was 0.0074 for PFOA and 0.061 for PFOS through the water intake, respectively, suggesting that the levels of PFOA and PFOS in Liaohe River would not result in the immediate health impact via water intake alone.

\section{Mass inventory of PFCs}

Riverine sink is one major process for the transportation and mobilization of pollutants to the sea. In this study, the potential of sediments as a PFCs source to the surrounding environment was assessed by the estimation of $\Sigma$ PFCs and PFOA mass inventories. The mass inventory was calculated by the equation $I=\sum k C_{\mathrm{i}} A_{\mathrm{i}} d \rho$, where $C_{\mathrm{i}}$ (ng/g) is the average sediment concentration of $\sum$ PFCs and PFOA, $A_{\mathrm{i}}$ is the area $\left(\mathrm{km}^{2}\right), d$ is the sediment thickness $(\mathrm{cm}), \rho$ is the bulk density of the dry sediment $(\mathrm{g} /$ $\mathrm{cm}^{2}$ ), and $k$ is the unit conversion factor. Assuming a depth of $20 \mathrm{~cm}$, a bulk density of $1.5 \mathrm{~g} / \mathrm{cm}^{3}$ and an area of $219,600 \mathrm{~km}^{2}$ [45], the mass inventories of 2 PFCs and PFOA were $328.74 \mathrm{t}$ and $103.43 \mathrm{t}$ in Liaohe River, respectively. A study conducted in Taihu Lake showed that the mass inventory of perfluoroalkyl acids in the lake was $989 \mathrm{~kg}$ [46], which was much higher than in the present study. However, the mass inventory of PFCs in Liaohe River was still able to contribute to the PFCs contamination in the coastal area.

\section{Conclusions}

This study demonstrated that PFCs were widely presented in the water and sediments of Liaohe River. In general, PFCAs had higher concentrations and detection frequency than PFSAs, and PFCAs with short carbon chains (PFBA, PFHxA, PFOA, PFHpA and PFNA) had much higher detection frequencies than long-carbon chain compounds (PFDA, PFUnDA, PFDoDA and PFTrDA) in water. Among all PFC homologues, the average $K_{\mathrm{oc}}$ increased with the increased carbon chain, by 0.13-0.62 log units with each $\mathrm{CF}_{2}$ moiety. $K_{\text {oc }}$ values were higher for PFSAs than PFCAs. The risk assessment suggested that PFOA in water may exert adverse effect on the aquatic ecosystem. The mass inventories of $\Sigma \mathrm{PFCs}$ and PFOA at $328.74 \mathrm{t}$ and $103.43 \mathrm{t}$ in the study area indicated that Liaohe River was likely acting as a PFCs source to the surrounding area.

\footnotetext{
Abbreviations

PFC: perfluorinated compounds; PFCAs: perfluorocarboxylic acids; PFASs: perfluoroalkyl sulfonates; PFBA: perfluorobutanoate; PFPA: perfluoropentanoate; PFHXA: perfluorohexanoate; PFHpA: perfluoroheptanoate; PFOA: perfluorooctanoic acid; PFNA: perfluorononanoate; PFDA: perfluorodecanoate; PFUnDA: perfluoroundecanoate; PFDoDA: perfluorododecanoate; PFTrDA: perfluorotridecanoate; PFBS: perfluorobutane sulfonate; PFOS: perfluorooctane sulfonate; PFDS: perfluorodecane sulfonate; PFHxA: potassium salts of perfluorohexane sulfonate; PFOSA: perfluorooctane sulfonamide; POSF: perfluorooctane
} 
sulfonyl fluoride; PP: polypropylene; PTFE: polytetrafluoroethene; BDL: below detection limit; LOD: limit of detection; LOQ: limit of quantification; S/N: signal/ noise ratios; $\mathrm{RQ}$ : risk quotient; $\mathrm{EQS}$ : environmental quality standards; $\mathrm{HR}$ : hazard ratio; RD: reference dose.

\section{Acknowledgements}

Not applicable.

\section{Authors' contributions}

$J \mathrm{~L}, C G$ and $J X$ involved in the experiments and manuscript writing, SL and $Y Z$ were responsible for the data analysis and study designing. $J L$ and $J X$ contributed to the manuscript correction. All authors read and approved the final manuscript.

\section{Funding}

This work was financially supported by Major Science and Technology Program for Water Pollution Control and Treatment (2017ZX07302001) and Free Exploration Foundation of State Key Laboratory of Environmental Criteria and Risk Assessment, Chinese Research Academy of Environmental Sciences.

\section{Availability of data and materials}

The datasets used and analyzed during the current study are available from the corresponding author on reasonable request.

\section{Ethics approval and consent to participate}

Not applicable.

\section{Consent for publication}

Not applicable.

\section{Competing interests}

The authors declare that they have no competing interests.

\section{Author details}

1 State Key Laboratory of Environmental Criteria and Risk Assessment, Chinese Research Academy of Environmental Sciences, Beijing 100012, China. ${ }^{2}$ Key Laboratory of Analytical Science and Technology of Hebei Province, College of Chemistry and Environmental Science, Hebei University, Baoding 071002, China.

Received: 4 July 2019 Accepted: 26 August 2019

Published online: 10 September 2019

\section{References}

1. Roos PH, Angerer J, Dieter H, Wilhelm M, Wölfle D, Hengstler JG (2008) Perfluorinated compounds (PFC) hit the headlines. Arch Toxicol 82(1):57-59

2. Kissa E (2001) Fluorinated surfactants and repellents. CRC Press, Boca Raton

3. Bao J, Jin Y, Liu W, Ran X, Zhang Z (2009) Perfluorinated compounds in sediments from the Daliao River system of northeast China. Chemosphere 77(5):652-657

4. Shoeib M, Vlahos P, Harner T, Peters A, Graustein M, Narayan J (2010) Survey of polyfluorinated chemicals (PFCs) in the atmosphere over the northeast Atlantic Ocean. Atmos Environ 44(24):2887-2893

5. Chen L, Tsui MM, Lam JC, Wang Q, Hu C, Wai OW, Zhou B, Lam PKJEP (2018) Contamination by perfluoroalkyl substances and microbial community structure in Pearl River Delta sediments. Environ Pollut 245:218-225

6. Codling G, Vogt A, Jones PD, Wang T, Wang P, Lu YL, Corcoran M, Bonina S, Li A, Sturchio NC (2014) Historical trends of inorganic and organic fluorine in sediments of Lake Michigan. Chemosphere 114:203-209

7. Stock NL, Furdui VI, Muir DCG, Mabury SA (2007) Perfluoroalkyl contaminants in the Canadian Arctic: evidence of atmospheric transport and local contamination. Environ Sci Technol 41(10):3529-3536

8. Becker AM, Gerstmann S, Frank H (2010) Perfluorooctanoic acid and perfluorooctane sulfonate in two fish species collected from the Roter Main River, Bayreuth, Germany. Bull Environ Contam Toxicol 84(1):132-135
9. So M, Taniyasu S, Lam P, Zheng G, Giesy J, Yamashita N (2006) Alkaline digestion and solid phase extraction method for perfluorinated compounds in mussels and oysters from South China and Japan. Arch Environ Contam Toxicol 50(2):240-248

10. Taniyasu S, Kannan K, Horii Y, Hanari N, Yamashita N (2003) A survey of perfluorooctane sulfonate and related perfluorinated organic compounds in water, fish, birds, and humans from Japan. Environ Sci Technol 37(12):2634-2639

11. Kannan K, Yun SH, Evans TJ (2005) Chlorinated, brominated, and perfluorinated contaminants in livers of polar bears from Alaska. Environ Sci Technol 39(23):9057-9063

12. Kärrman A, Ericson I, van Bavel B, Darnerud PO, Aune M, Glynn A, Lignell S, Lindström G (2007) Exposure of perfluorinated chemicals through lactation: levels of matched human milk and serum and a temporal trend, 1996-2004, in Sweden. Environ Health Perspect 115:226-230

13. Houde M, Bujas TA, Small J, Wells RS, Fair PA, Bossart GD, Solomon KR, Muir DC (2006) Biomagnification of perfluoroalkyl compounds in the bottlenose dolphin (Tursiops truncatus) food web. Environ Sci Technol 40(13):4138-4144

14. Lim TC, Wang B, Huang J, Deng S, Yu G (2011) Emission inventory for PFOS in China: review of past methodologies and suggestions. Sci World J 11:1963-1980

15. Xie S, Wang T, Liu S, Jones KC, Sweetman AJ, Lu Y (2006) Industrial source identification and emission estimation of perfluorooctane sulfonate in China. Environ Int 52:1-8

16. Hansen KJ, Johnson H, Eldridge J, Butenhoff J, Dick L (2002) Quantitative characterization of trace levels of PFOS and PFOA in the Tennessee River. Environ Sci Technol 36(8):1681-1685

17. Prevedouros K, Cousins IT, Buck RC, Korzeniowski SH (2006) Sources, fate and transport of perfluorocarboxylates. Environ Sci Technol 40(1):32-44

18. Sinclair E, Kannan K (2006) Mass loading and fate of perfluoroalkyl surfactants in wastewater treatment plants. Environ Sci Technol 40(5):1408-1414

19. Murakami M, Shinohara H, Takada H (2009) Evaluation of wastewater and street runoff as sources of perfluorinated surfactants (PFSs). Chemosphere 74(4):487-493

20. Guo W, He M, Yang Z, Lin C, Quan X, Wang H (2007) Distribution of polycyclic aromatic hydrocarbons in water, suspended particulate matter and sediment from Daliao River watershed, China. Chemosphere 68(1):93-104

21. Zhao X, Zhang H, Ni Y, Lu X, Zhang X, Su F, Fan J, Guan D, Chen J (2011) Polybrominated diphenyl ethers in sediments of the Daliao River Estuary, China: levels, distribution and their influencing factors. Chemosphere 82(9):1262-1267

22. Guo W, He M, Yang Z, Lin C, Quan X (2011) Aliphatic and polycyclic aromatic hydrocarbons in the Xihe River, an urban river in China's Shenyang City: distribution and risk assessment. J Hazard Mater 186(2):1193-1199

23. LV J, Xu J, Guo C, Zhang Y, Bai Y, Meng W (2014) Spatial and temporal distribution of polycyclic aromatic hydrocarbons (PAHs) in surface water from Liaohe River Basin, northeast China. Environ Sci Pollut Res 21(11):7088-7096

24. Wang HZ, He MC, Lin CY, Quan XC, Guo W, Yang ZF (2007) Monitoring and assessment of persistent organochlorine residues in sediments from the Daliaohe River Watershed, Northeast of China. Environ Monit Assess 133(1-3):231-242

25. Zhang H, Zhao X, Ni Y, Lu X, Chen J, Su F, Zhao L, Zhang N, Zhang X (2010) PCDD/Fs and PCBs in sediments of the Liaohe River, China: levels, distribution, and possible sources. Chemosphere 79(7):754-762

26. Gao Y, Zhang H, Su F, Tian Y, Chen J (2012) Environmental occurrence and distribution of short chain chlorinated paraffins in sediments and soils from the Liaohe River Basin, PR China. Environ Sci Technol 46(7):3771-3778

27. Xu J, Guo CS, Zhang Y, Meng W (2014) Bioaccumulation and trophic transfer of perfluorinated compounds in a eutrophic freshwater food web. Environ Pollut 184(1):254-261

28. Sun H, Li F, Zhang T, Zhang X, He N, Song Q, Zhao L, Sun L, Sun T (2011) Perfluorinated compounds in surface waters and WWTPs in Shenyang, China: mass flows and source analysis. Water Res 45(15):4483-4490

29. Dinglasan MJA, Ye Y, Edwards EA, Mabury SA (2004) Fluorotelomer alcohol biodegradation yields poly-and perfluorinated acids. Environ Sci Technol 38(10):2857-2864 
30. Pan CG, Ying GG, Liu YS, Zhang QQ, Chen ZF, Peng FJ, Huang GY (2014) Contamination profiles of perfluoroalkyl substances in five typical rivers of the Pearl River Delta region, South China. Chemosphere 114(22):16-25

31. Wang P, Wang T, Giesy JP, Lu Y (2013) Perfluorinated compounds in soils from Liaodong Bay with concentrated fluorine industry parks in China. Chemosphere 91(6):751-757

32. Li F, Sun H, Hao Z, He N, Zhao L, Zhang T, Sun T (2011) Perfluorinated compounds in Haihe River and Dagu drainage canal in Tianjin, China. Chemosphere 84(2):265-271

33. Pan G, You C (2010) Sediment-water distribution of perfluorooctane sulfonate (PFOS) in Yangtze River Estuary. Environ Pollut 158(5):1363-1367

34. Kwadijk C, Korytar P, Koelmans A (2010) Distribution of perfluorinated compounds in aquatic systems in The Netherlands. Environ Sci Technol 44(10):3746-3751

35. Zhou Z, Liang Y, Shi Y, Xu L, Cai Y (2013) Occurrence and transport of perfluoroalkyl acids (PFAAs), including short-chain PFAAs in Tangxun Lake, China. Environ Sci Technol 47(16):9249-9257

36. Lutz A, Nobuyoshi Y, Yeung LWY, Sachi T, Yuichi H, Lam PKS, Ralf E (2009) Partitioning behavior of per- and polyfluoroalkyl compounds between pore water and sediment in two sediment cores from Tokyo Bay, Japan. Environ Sci Technol 43(18):6969-6975

37. Higgins CP, Luthy RG (2006) Sorption of perfluorinated surfactants on sediments. Environ Sci Technol 40(23):7251-7256

38. Wang T, Khim JS, Chen C, Naile JE, Lu Y, Kannan K, Park J, Luo W, Jiao W, Hu W (2012) Perfluorinated compounds in surface waters from Northern China: comparison to level of industrialization. Environ Int 42:37-46

39. Zhang W, Zhang Y, Taniyasu S, Yeung LW, Lam PK, Wang J, Li X, Yamashita N, Dai J (2013) Distribution and fate of perfluoroalkyl substances in municipal wastewater treatment plants in economically developed areas of China. Environ Pollut 176:10-17
40. Simcik MF, Dorweiler KJ (2006) Ratio of perfluorochemical concentrations as a tracer of atmospheric depositionto surface waters. Environ Sci Technol 40(1):410

41. Armitage JM, Matthew M, Cousins IT (2009) Comparative assessment of the global fate and transport pathways of long-chain perfluorocarboxylic acids (PFCAs) and perfluorocarboxylates (PFCs) emitted from direct sources. Environ Sci Technol 43(15):5830-5836

42. Valsecchi S, Conti D, Crebelli R, Polesello S, Rusconi M, Mazzoni M, Preziosi E, Carere M, Lucentini L, Ferretti E (2017) Deriving environmental quality standards for perfluorooctanoic acid (PFOA) and related short chain perfluorinated alkyl acids. J Hazard Mater 323:84-98

43. Brooke D, Footitt A, Nwaogu TJEA (2004) Environmental risk evaluation report: perfluorooctanesulphonate (PFOS). UK Environment Agency, London

44. Hoke RA, Bouchelle LD, Ferrell BD, Buck RCJC (2012) Comparative acute freshwater hazard assessment and preliminary PNEC development for eight fluorinated acids. Chemosphere 87(7):725-733

45. Lv J (2015) Pollution characteristics of representative persistent organic pollutants in the aquatic environment of Liaohe River. Chinese Research Academy of Environmental Sciences, Beijing

46. Guo C, Zhang Y, Zhao X, Du P, Liu S, Lv J, Xu F, Meng W, Xu J (2015) Distribution, source characterization and inventory of perfluoroalkyl substances in Taihu Lake, China. Chemosphere 127:201-207

\section{Publisher's Note}

Springer Nature remains neutral with regard to jurisdictional claims in published maps and institutional affiliations.

\section{Submit your manuscript to a SpringerOpen ${ }^{\odot}$ journal and benefit from:}

- Convenient online submission

- Rigorous peer review

- Open access: articles freely available online

- High visibility within the field

- Retaining the copyright to your article

Submit your next manuscript at $\boldsymbol{\nabla}$ springeropen.com 